\title{
Lixiviación de la calcopirita en medios $\operatorname{clorurados}^{(\cdot)}$
}

\author{
T. Ibáñez* y L. Velásquez
}

Resumen

El objetivo de esta investigación es determinar el efecto de parámetros y aditivos $\left(\mathrm{MnO}_{2}\right.$ - iones de plata - pirita) en la cinética de disolución de la calcopirita mediante pruebas de disolución con concentrado de calcopirita y calcopirita natural en matraces y reactores agitados mecánicamente bajo condiciones moderadas. La disolución de la calcopirita en medios clorurados es dependiente del potencial de la solución, obteniéndose valores de velocidad aceptables dentro de los 540 y $630 \mathrm{mV}$ (SHE). El pH alrededor de los 2,5 permite mantener los potenciales de solución en valores donde la calcopirita lixivia a velocidades aceptables debido a que ocurre la precipitación de hierro. Tanto la pirita como los iones de plata catalizan la disolución y el efecto es mayor con ambas especies presentes. $\mathrm{El} \mathrm{MnO}_{2}$ posee un efecto negativo en la disolución debido a que aumenta el potencial de la solución a valores donde la velocidad disminuye considerablemente.

Palabras clave Lixiviación de calcopirita; Medios clorurados; Potencial de solución; Aditivos catalizadores; $\mathrm{MnO}_{2}$; Pirita; Iones de plata.

\section{The dissolution of chalcopyrite in chloride media}

\begin{abstract}
The aim of this investigation is to determinate the effects of parameters and additives on the kinetics of dissolution of chalcopyrite on moderated conditions by means of dissolutions test with chalcopyrite concentrate and pure chalcopyrite in shake flasks and instrumented stirred reactors. A study of the dissolution of chalcopyrite in chloride solutions has demonstrated that the rate of dissolution of chalcopyrite is strongly dependent on the potential of the solution within a range of 540 to $630 \mathrm{mV}$ (versus SHE). Leaching at $\mathrm{pH}$ around 2.5 results in increased rates of copper dissolution suggesting the possibility to keep the solution potential within the range. Both pyrite and silver ions enhance the dissolution of chalcopyrite and this effect increases when both species are present. The $\mathrm{MnO}_{2}$ has a negative effect on the dissolution increasing the solution potential to values where the rate decreases considerably.
\end{abstract}

Keywords

Dissolution of chalcopyrite; Chloride media; Solution potential; Catalysts additives; $\mathrm{MnO}_{2}$; Pyrite; Silver ions.

\section{INTRODUCCIÓN}

Los procesos hidrometalúrgicos, como las pilas de lixiviación (LX), seguidos por los procesos de extracción por solventes (SX) y electro-obtención (EW) son aplicados a óxidos y sulfuros secundarios de cobre. Sin embargo, estos óxidos y sulfuros secundarios se encuentran en la superficie de los depósitos, los cuales se están agotando, y sólo van quedando los sulfuros primarios. Debido a esto, pronto las faenas mineras deberán enfrentar la problemática del agotamiento de material de alimentación a las plantas de LX-SX-EW, además de leyes cada vez más bajas, escasez del recurso hídrico y que la única alimentación disponible a futuro será la calcopirita, sulfuro de cobre cuyo tradicional procesamiento es a través de una combina- ción de técnicas de flotación y pirometalúrgicas. La calcopirita es el mineral más refractario y abundante de los sulfuros de cobre ${ }^{[1 \text { y } 2]}$, por lo que la metalurgia extractiva de este metal se basa principalmente en este mineral ${ }^{[3]}$. Debido a esto, para permitir el uso continuo de estas plantas, es necesario desarrollar alternativas tecnológicas para el procesamiento de estos sulfuros abordando de esta forma la lenta cinética de lixiviación del principal sulfuro primario de cobre.

Es por esto que existen varios estudios relacionados con la lixiviación de este mineral en diferentes medios, de los cuales los más comunes son amoniacales, nitratados, sulfatados, clorurados y la utilización de bacterias. De ellos, la ventaja de utilizar medios clorurados es que es uno de los principales compo-

(•) Trabajo recibido el día 16 de marzo de 2012 y aceptado en su forma final el día 11 de enero de 2013.

Departamento de Ingeniería Metalúrgica, Universidad Católica del Norte, Antofagasta, Chile. 
nentes del agua de mar, conteniendo aproximadamente $20 \mathrm{~g} / \mathrm{L}$ de iones cloruros ${ }^{[4]} \mathrm{y}$, debido a la escasez de agua en el norte de Chile, las empresas mineras se están planteando la posibilidad de utilizar agua de mar en su proceso.

Son varias las investigaciones relacionadas con la disolución de la calcopirita pero aún no existe un acuerdo general en ciertos aspectos importantes, los cuales nos pueden llevar a un mejor entendimiento del proceso y así a un escalamiento a nivel industrial. En el presente trabajo se investigó la disolución de la calcopirita en medios clorurados a presión atmosférica y temperaturas moderadas con el propósito de desarrollar un proceso que puede ser utilizado en la industria minera del cobre.

\section{METODOLOGÍA EXPERIMENTAL}

Se realizaron pruebas de lixiviación en matraces y reactores agitados mecánicamente con concentrado de calcopirita y calcopirita natural: el concentrado de calcopirita con una granulometría de $-38+25 \mu \mathrm{m}$ (malla Ty) y la calcopirita natural con $\mathrm{P}_{80}$ de $28,4 \mu \mathrm{m}$ según los resultados entregados por Microtrac. Los análisis de QEMSCAN de las muestras se encuentran en la tabla I.

Tabla I. Resultados obtenidos mediante análisis QEMSCAN para las muestras de calcopirita utilizadas

Table I. QEMSCAN mineralogical composition of chalcopyrite

\begin{tabular}{lcr}
\hline $\begin{array}{l}\text { Porcentajes en } \\
\text { peso de especies }\end{array}$ & $\begin{array}{r}\text { Concentrado } \\
\text { de calcopirita }\end{array}$ & $\begin{array}{r}\text { Calcopirita } \\
\text { natural }\end{array}$ \\
\hline Calcopirita & 72,98 & 85,39 \\
Pirita & 10,10 & 10,41 \\
Otros sulfuros de Cu & 4,11 & 2,39 \\
Cobre & 26,29 & 31,24 \\
Hierro & 28,77 & 31,43 \\
\hline
\end{tabular}

Las pruebas en matraces $(250 \mathrm{~mL})$ se realizaron en un agitador termocalefactor a $50^{\circ} \mathrm{C}, 140 \mathrm{rpm}$, con calcopirita natural y 40 días de duración. En ellas se evaluaron la presencia de aditivos cuyas soluciones de lixiviación contenían un volumen de $100 \mathrm{~mL}$,

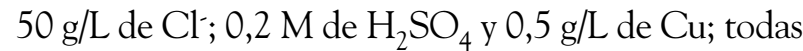
fueron preparadas utilizando agua destilada. Las proporciones de masa evaluadas para la pirita y $\mathrm{MnO}_{2}$ en función de la masa de calcopirita adicionada $(1,11 \mathrm{~g})$ fueron $1: 1 ; 1: 3$ y $1: 5$ y para el caso de los iones de plata 1,2 y 5 ppm con la misma cantidad de calcopirita de los otros aditivos. Todas las pruebas en matraces agitados se cubrieron con algodón para disminuir las pérdidas por evaporación y permitir el acceso de oxígeno a la solución. Además, se adicionó agua destilada para mantener el volumen inicial de pulpa debido a la evaporación de agua. Se tomaron muestras líquidas y se enviaron a análisis químico (previamente filtradas) por $\mathrm{Cu}_{\text {total }}, \mathrm{Fe}_{\text {total }}$ y $\mathrm{Mn}$ o $\mathrm{Ag}$ según correspondiera, reponiendo siempre una solución de igual volumen a la retirada y de la misma concentración que la solución inicial.

Los reactores mecánicamente agitados son cilíndricos y de vidrio de un volumen de $1000 \mathrm{~mL}$ y se cubren con una tapa multipuerto de PVC donde está montado un motor con un agitador de titanio y además es posible controlar la temperatura en el reactor. Las pruebas realizadas en estos equipos fueron con concentrado de calcopirita y se evaluaron $\mathrm{pH}(\mathrm{pH}<2$ y $\mathrm{pH}$ aproximado a 2,5$)$ y temperatura $\left(35,50,75\right.$ y $\left.90^{\circ} \mathrm{C}\right)$. Los procedimientos fueron idénticos a los mencionados en las pruebas en matraces agitados.

Los reactivos utilizados fueron cloruro de sodio, ácido sulfúrico y sulfato de cobre penta-hidratado, todos de grado P.A y de la marca Merck. Los aditivos utilizados fueron un estándar de plata para AAS de $1000 \mathrm{mg} / \mathrm{L}$, óxido de manganeso (IV) sintético de una pureza $99,98 \%$ y pirita natural proveniente de la mina Navajún con una pureza de 98,84 \% (Análisis QEMSCAN).

\section{RESULTADOS Y DISCUSIONES}

\subsection{Efecto del pH}

En la figura 1 se observan los resultados para dos pruebas, la primera realizada a $\mathrm{pH}<2$ y la segunda a $\mathrm{pH}$ alrededor de 2,5. A partir de ella se puede decir que a 2,5 la cinética de disolución del concentrado de calcopirita es alta $(99,31 \%)$ y esto probablemente se debe a los potenciales de solución alcanzados, entre 540-630 mV (SHE). Sobre los $630 \mathrm{mV}$ la velocidad de disolución comienza a disminuir. Al contrario, para la prueba con $\mathrm{pH}<2$ los potenciales de solución se encuentran sobre los $660 \mathrm{mV}$, por lo que la extracción de cobre es baja (14,06 \%). Esto confirmaría la dependencia de la disolución de este sulfuro primario con los potenciales de la solución. Los resultados obtenidos tendrían relación con lo reportado por otros autores ${ }^{[5]}$, quienes mencionan la existencia de un rango de potencial donde la calcopirita lixivia a 


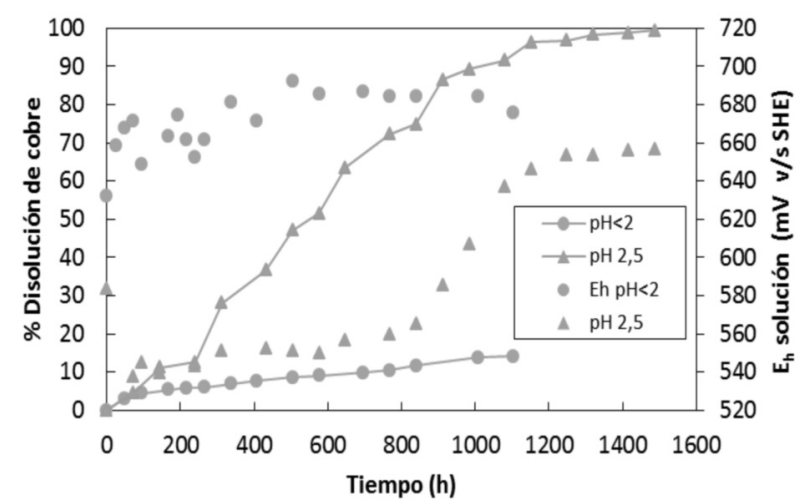

Figura 1. Disolución de cobre a partir del concentrado de calcopirita $(-38+25 \mu \mathrm{m})$ a $50{ }^{\circ} \mathrm{C}$; $50 \mathrm{~g} / \mathrm{L} \mathrm{de} \mathrm{Cl}^{-} ; 0,5 \mathrm{~g} / \mathrm{L}$ de $\mathrm{Cu}^{2+}$ a pH $<2$ y pH 2,5 en reactores mecánicamente agitados.

Figure 1. Copper dissolution from chalcopyrite concentrate $(-38+25 \mu \mathrm{m})$ at $50^{\circ} \mathrm{C} ; 50 \mathrm{~g} / \mathrm{L} \mathrm{of} \mathrm{Cl}^{-}$; $0.5 \mathrm{~g} / \mathrm{L}$ of $\mathrm{Cu}^{2+}$ at $\mathrm{pH}<2$ and $\mathrm{pH} 2.5$ in a instrumented stirred reactor.

velocidades aceptables que se encontraría entre los 560-620 mV (SHE).

Probablemente debido a que existió la precipitación de hierro en la prueba a $\mathrm{pH} 2,5$, los valores de potenciales de solución alcanzados son más bajos en comparación a la prueba a $\mathrm{pH}<2$. Esta situación se puede corroborar con la tonalidad de los residuos de lixiviación la cual es típica de los precipitados de hierro (color café rojizo), y además con la figura 2 donde se observa que a $\mathrm{pH}$ 2,5 no existe disolución de hierro

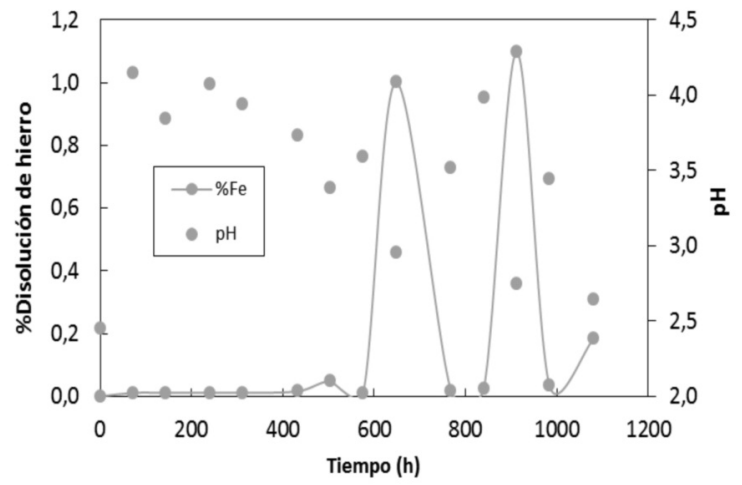

Figura 2. Disolución de hierro a partir del concentrado de calcopirita $(-38+25 \mu \mathrm{m})$ a $50{ }^{\circ} \mathrm{C}$; $50 \mathrm{~g} / \mathrm{L} \mathrm{de} \mathrm{Cl}^{-} ; 0,5 \mathrm{~g} / \mathrm{L}$ de $\mathrm{Cu}^{2+}$ a pH 2,5 en reactores mecánicamente agitados.

Figure 2. Iron dissolution from chalcopyrite concentrate $(-38+25 \mu \mathrm{m})$ at $50^{\circ} \mathrm{C} ; 50 \mathrm{~g} / \mathrm{L} \mathrm{of} \mathrm{Cl}^{-}$; $0.5 \mathrm{~g} / \mathrm{L}$ of $\mathrm{Cu}^{2+}$ at $\mathrm{pH} 2.5$ in a instrumented stirred reactors.

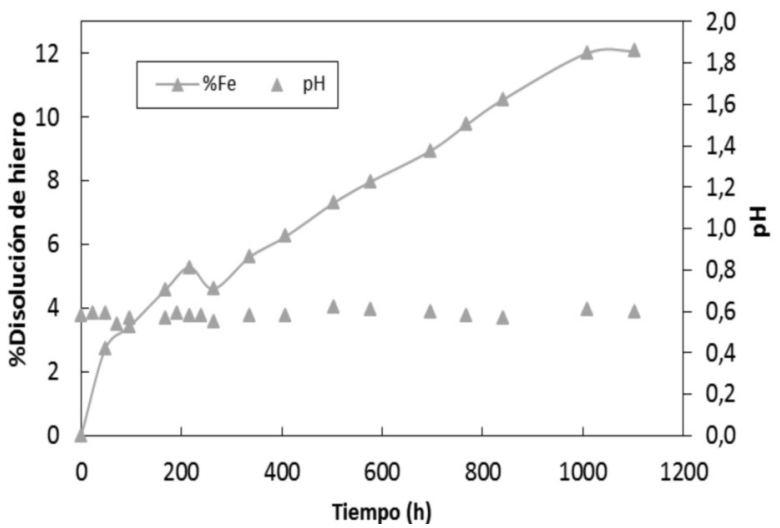

Figura 3. Disolución de hierro a partir del concentrado de calcopirita $(-38+25 \mu \mathrm{m})$ a $50^{\circ} \mathrm{C}$; $50 \mathrm{~g} / \mathrm{L}$ de $\mathrm{Cl}^{-} ; 0,5 \mathrm{~g} / \mathrm{L}$ de $\mathrm{Cu}^{2+}$ a $\mathrm{pH}<2$ en reactores mecánicamente agitados.

Figure 3. Iron dissolution from chalcopyrite concentrate $(-38+25 \mu \mathrm{m})$ at $50^{\circ} \mathrm{C} ; 50 \mathrm{~g} / \mathrm{L}$ of $\mathrm{Cr}^{-}$; $0.5 \mathrm{~g} / \mathrm{L}$ of $\mathrm{Cu}^{2+}$ at $\mathrm{pH}<2$ in a instrumented stirred reactors.

excepto cuando éste alcanza valores cercanos a 2 . Caso contrario para la prueba realizada a $\mathrm{pH}<2$ (Fig. 3). Por lo tanto, mantener el $\mathrm{pH}$ en alrededor de 2,5 ayudaría a mantener el rango de potencial donde la disolución de la calcopirita aumenta. Además, cabe mencionar que a pesar de la presencia de estos precipitados de hierro la velocidad de disolución no disminuyó, por lo que bajo estas condiciones no se podría asegurar que la presencia de estas especies sea la responsable de detener la disolución de la calcopirita como aseguran otros auto$\operatorname{res}^{[2 y 6]}$.

\subsection{Efecto de la temperatura}

Existe un acuerdo general de varios autores según el cual el efecto de la temperatura en la cinética de disolución de la calcopirita es significativo ${ }^{[7}$ y 8$]$. Al aumentar la temperatura se mejora la velocidad de disolución. En la figura 4 se presenta el efecto de la temperatura en la cinética de disolución de cobre del concentrado de calcopirita $(-38+25 \mu \mathrm{m})$ obtenido para este trabajo. A partir de ella se puede observar que existe una tendencia aproximadamente lineal del porcentaje de extracción de cobre con el tiempo y además un efecto positivo de esta variable, excepto a $90^{\circ} \mathrm{C}$, bajo las condiciones utilizadas ( $50 \mathrm{~g} / \mathrm{L} \mathrm{Cl}^{-} ; 0,5 \mathrm{~g} / \mathrm{L} \mathrm{Cu}^{2+}$ y $\left.0,2 \mathrm{M} \mathrm{H}_{2} \mathrm{SO}_{4}\right)$. La disminución de la disolución de cobre al aumentar la temperatura a $90^{\circ} \mathrm{C}$ se asocia a que bajo estas condiciones las partículas del concen- 


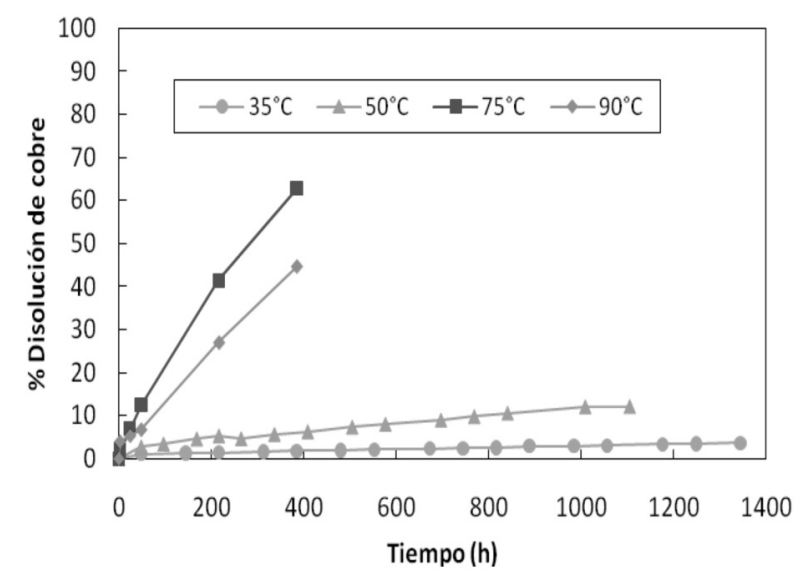

Figura 4. Efecto de la temperatura en la disolución del concentrado de calcopirita $(-38+25 \mu \mathrm{m})$

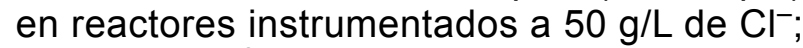

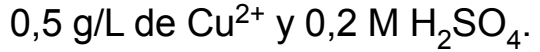

Figure 4. The effect of temperature on the dissolution of chalcopyrite concentrate $(-38+25 \mu \mathrm{m})$ at $50 \mathrm{~g} / \mathrm{L}$ of $\mathrm{Cl}^{-} ; 0.5 \mathrm{~g} / \mathrm{L}$ of $\mathrm{Cu}^{2+}$ and $0.2 \mathrm{M} \mathrm{H}_{2} \mathrm{SO}_{4}$ in a mechanically stirred reactors.

trado sufrieron una aglomeración, por lo que se cree que disminuyó la superficie de contacto con los reactantes.

A partir de la información suministrada en la figura 4 se determinó la pendiente de cada prueba, la cual corresponde al valor de la constante cinética $\left(\mathrm{K}\left(\mathrm{s}^{-}\right)\right)$. Luego se graficó el diagrama de Arrhenius (Fig. 5) a través del cual se obtuvo el valor de la energía de activación del concentrado de calcopirita, el cual es $96,55 \mathrm{~kJ} / \mathrm{mol}$ entre 35 y $75^{\circ} \mathrm{C}$, y esto nos indica que la velocidad de disolución, bajo las condiciones estudiadas, es controlada por la reacción química.

\section{TERMODINÁMICA DE LA DISOLUCIÓN DE COBRE EN MEDIOS CLORURADOS A $50^{\circ} \mathrm{C}$}

\subsection{Diagrama $E_{h}$ versus concentración de cloruro}

Para que ocurra la oxidación espontánea de un mineral se requiere que el potencial del par oxidante sea mayor que el de la oxidación de tal mineral. Según la figura 6, la oxidación de la calcopirita ocurre a cualquier concentración de iones cloruros, tanto para el par $\mathrm{Cu}^{2+} / \mathrm{Cu}^{+}$como para $\mathrm{Fe}^{3+} / \mathrm{Fe}^{2+}$, siendo el oxidante más fuerte el par $\mathrm{Cu}^{2+} / \mathrm{Cu}^{+}$y por debajo de esta concentración el par $\mathrm{Fe}^{3+} / \mathrm{Fe}^{2+}$. Este

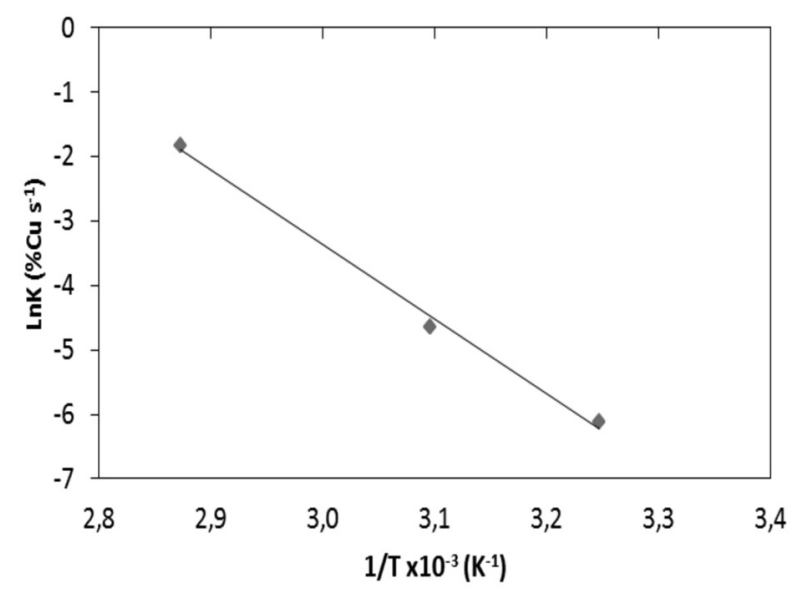

Figura 5. Gráfico de Arrhenius para la disolución de cobre desde el concentrado de calcopirita $(-38+25 \mu \mathrm{m})$ entre 35 y $70{ }^{\circ} \mathrm{C}$ en reactores ins-

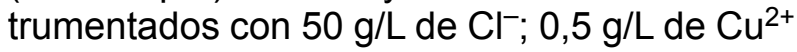
y $0,2 \mathrm{M} \mathrm{H}_{2} \mathrm{SO}_{4}$.

Figure 5. Arrhenius plot for the dissolution of chalcopyrite concentrate $(-38+25 \mu \mathrm{m})$ between 35 and $70{ }^{\circ} \mathrm{C}$ at $50 \mathrm{~g} / \mathrm{L}$ of $\mathrm{Cl}^{-} ; 0.5 \mathrm{~g} / \mathrm{L}$ of $\mathrm{Cu}^{2+}$ and $0.2 \mathrm{M} \mathrm{H}_{2} \mathrm{SO}_{4}$ in a mechanically stirred reactors.

mismo análisis es posible realizarlo para cualquier reacción presentada en la figura 6 .

La curva del par $\mathrm{Cu}^{2+} / \mathrm{Cu}^{+}$aumenta al aumentar la concentración de iones cloruros debido a que los iones cuprosos forman complejos fuertes con los iones cloruros, entonces su concentración disminuye y por ende los potenciales aumentan. Situación contraria ocurre con $\mathrm{Fe}^{3+} / \mathrm{Fe}^{2+}$ ya que es el ión férrico el que forma complejos fuertes con los iones cloruros, disminuyendo así los valores de potencial al aumentar la concentración de cloruro. Esta situación se observará en los diagramas de especies presentados en la sección siguiente.

\subsection{Diagrama de especies}

A partir de las figuras 7, 8 y 9 se realizaron los siguientes análisis termodinámicos:

Todo el $\mathrm{Cu}(\mathrm{I})$ se encuentra formando complejos con los iones cloruro de la forma $\mathrm{CuCl}_{2}{ }^{-}$y $\mathrm{CuCl}_{3}{ }^{-2}$ (Fig. 7), los cuales son las especies predominantes dentro del rango de concentraciones de cloruro estudiadas (0 - $100 \mathrm{~g} / \mathrm{L})$. Para el caso del sistema $\mathrm{Cu}(\mathrm{II})$-cloruro se observa que las especies predominantes son $\mathrm{CuCl}^{+}$ y $\mathrm{Cu}^{2+}$ (Fig. 8).

En el caso del hierro, casi todo el Fe(III) se encuentra como $\mathrm{FeCl}^{2+}$ y sólo a bajas concentraciones 


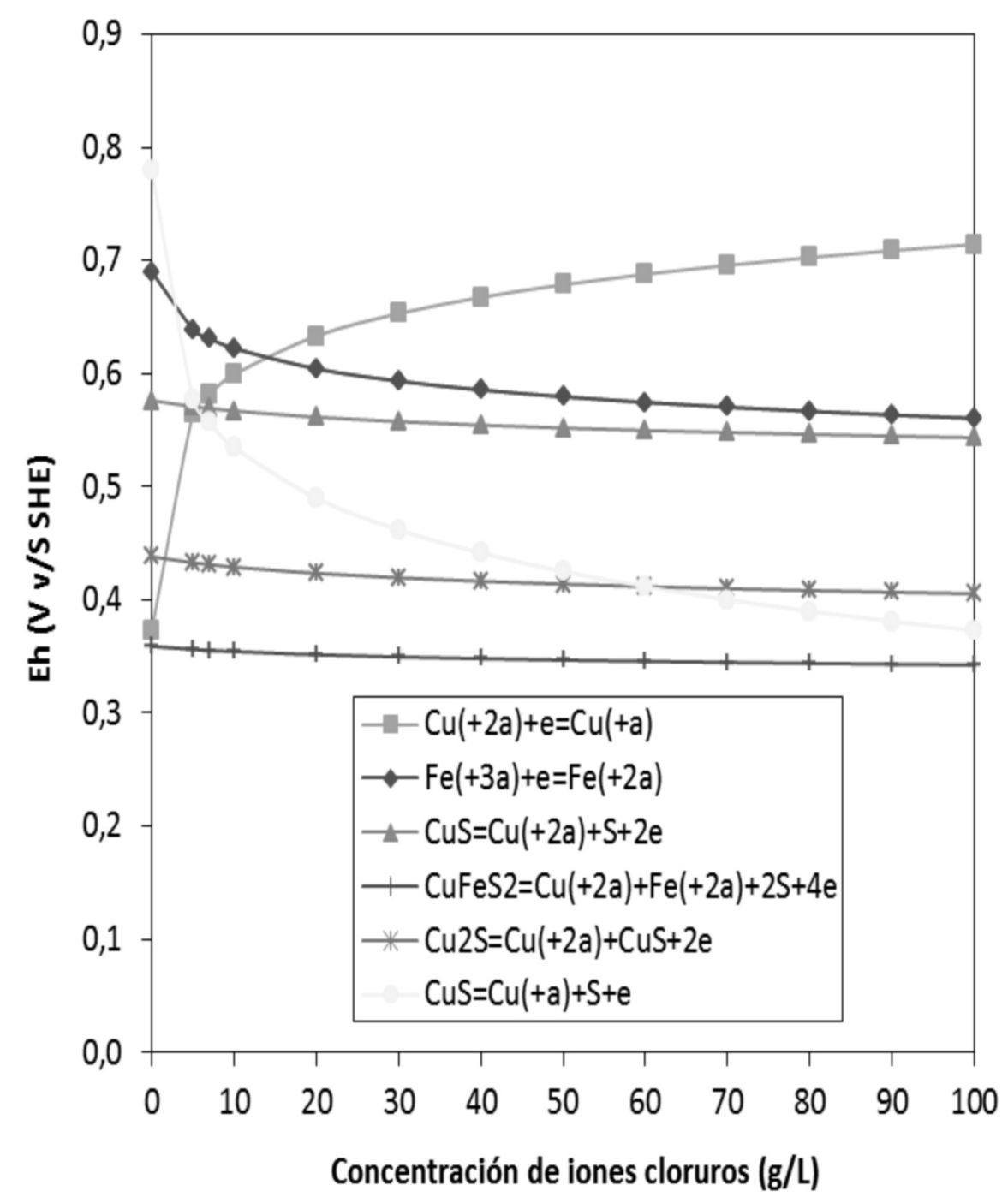

Figura 6. Diagrama de potencial de solución versus la concentración de iones cloruros de varias reacciones redox a $50{ }^{\circ} \mathrm{C} ; 1,08 \mathrm{~g} / \mathrm{L} \mathrm{Cu_{ \text {Total } }}$ y $0,51 \mathrm{~g} / \mathrm{L}$ de $\mathrm{Fe}_{\text {Total }}$.

Figure 6. Eh versus chloride concentration of several redox reactions

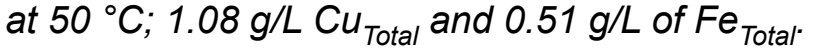

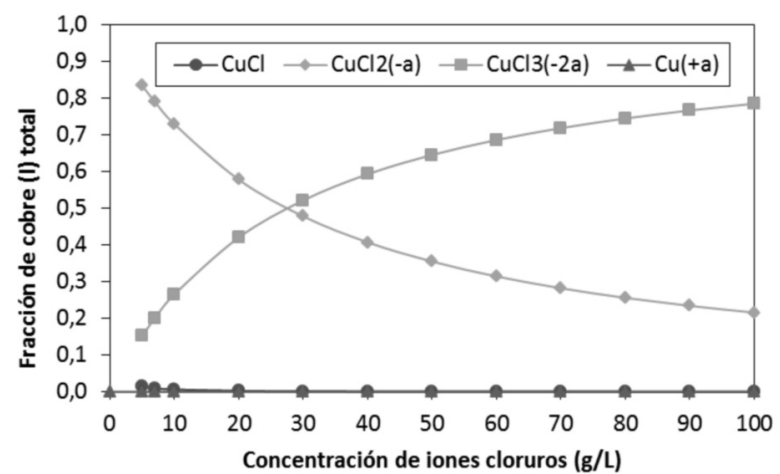

Figura 7. Diagrama de especies para el sistema $\mathrm{Cu}(\mathrm{I})-\mathrm{Cl}^{-}$a $50^{\circ} \mathrm{C}$.

Figure 7. Species distribution $\mathrm{Cu}(\mathrm{I})-\mathrm{Cl}^{-}$system at $50^{\circ} \mathrm{C}$.

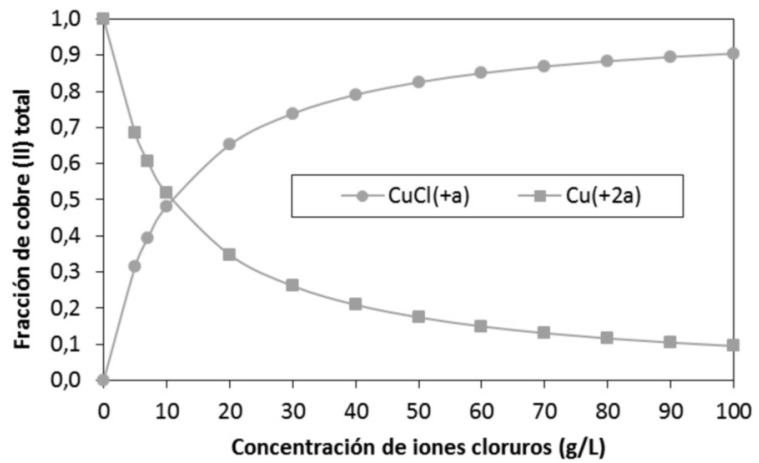

Figura 8. Diagrama de especies para el sistema $\mathrm{Cu}(\mathrm{II})-\mathrm{Cl}^{-}$a $50^{\circ} \mathrm{C}$.

Figure 8. Species distribution $\mathrm{Cu}(\mathrm{II})-\mathrm{Cl}^{-}$system at $50^{\circ} \mathrm{C}$. 


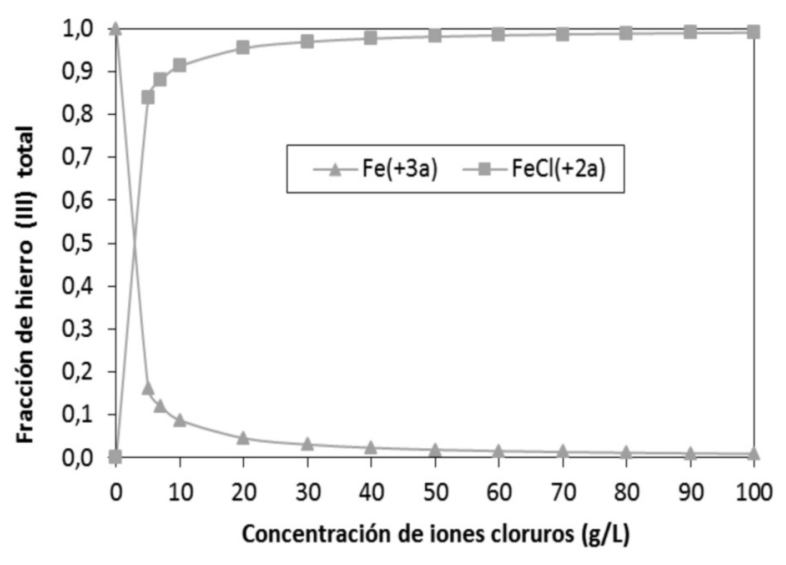

Figura 9. Diagrama de especies para el sistema $\mathrm{Fe}(\mathrm{III})-\mathrm{Cl}^{-}$a $50^{\circ} \mathrm{C}$.

\section{Figure 9. Species distribution $\mathrm{Fe}(\mathrm{III})-\mathrm{Cl}^{-}$system} at $50^{\circ} \mathrm{C}$.

de iones cloruros podemos encontrar los iones $\mathrm{Fe}^{3+}$ (Fig. 9).

De acuerdo a la información termodinámica presentada, se puede decir que los iones $\mathrm{Cu}^{+}$son estabilizados en soluciones cloruradas formando complejos con los iones cloruros, como los mencionados, por lo que no existe $\mathrm{Cu}^{+}$disponible en solución. Debido a que los iones cloruros están formando iones complejos fuertes con los $\mathrm{Cu}^{+}$, se observa que a $50 \mathrm{~g} / \mathrm{L}$ de cloruro existe $\mathrm{Cu}^{2+}$ disponible para ser utilizado en la disolución de la calcopirita.

Se puede decir que bajo las condiciones utilizadas en este trabajo no es posible que los iones férricos sean los oxidantes ya que a $50 \mathrm{~g} / \mathrm{L}$ de iones cloruros casi todo el $\mathrm{Fe}(\mathrm{III})$ se encuentra de la forma $\mathrm{FeCl}^{2+}$, por lo que casi no existe $\mathrm{Fe}^{3+}$ disponible para la reacción. Además, cabe mencionar que la presencia de este ión eleva los potenciales de solución a valores donde, por lo que observamos en las pruebas de lixiviación presentadas anteriormente, la disolución de la calcopirita disminuye considerablemente.

\section{EFECTO DE ADITIVOS}

\subsection{Efecto de la pirita}

La presencia de pirita en la cinética de disolución de muestras de calcopirita natural posee un efecto catalizador como se observa en la figura 10. El aumento de la concentración de pirita en el sistema aumenta la disolución de cobre. La presencia de pirita y plata (en una razón 1:5 de calcopirita:pirita y $5 \mathrm{mg} / \mathrm{L}$ de iones de plata), mejora aún más la disolución de

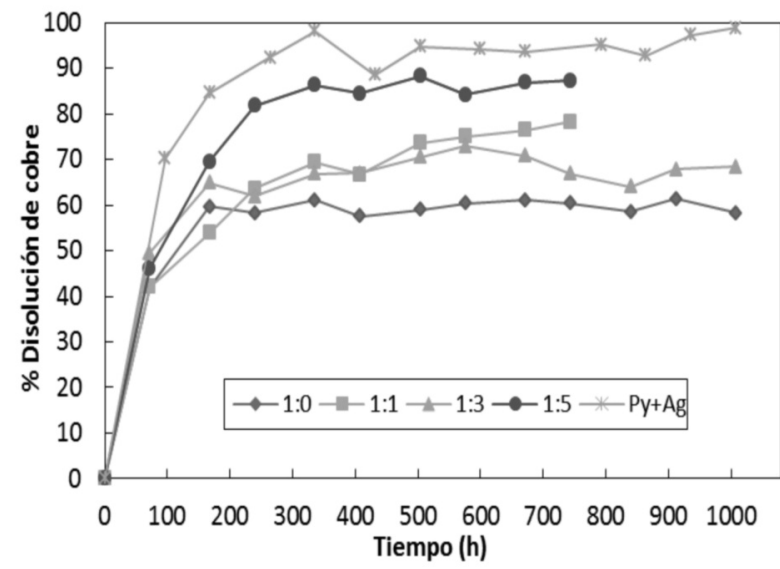

Figura 10. Disolución de cobre a partir de pruebas realizadas con calcopirita natural en presencia de distintas concentraciones de pirita realizadas en matraces agitados a $50{ }^{\circ} \mathrm{C} ; 50 \mathrm{~g} / \mathrm{L}$ de $\mathrm{Cl}^{-} ; 0,5 \mathrm{~g} / \mathrm{L}$ de $\mathrm{Cu}^{2+}$ y $0,2 \mathrm{M} \mathrm{H}_{2} \mathrm{SO}_{4}$.

Figure 10. Copper dissolution from natural chalcopyrite with pyrite presence in flaks at $50^{\circ} \mathrm{C}$;

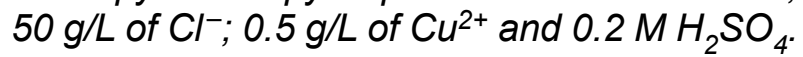

cobre. Esto está de acuerdo con lo publicado por otros autores ${ }^{[9]}$ en estudios realizados en medios de sulfato férrico.

Esto también se puede relacionar con los potenciales de solución, ya que al aumentar la cantidad de pirita disminuye el potencial de solución (Fig. 11) y, como se observó en las pruebas anteriores, la calcopirita lixivia a mejores cinéticas en potenciales de solución dentro de un rango, 540-630 mV, mientras que sobre estos valores la velocidad disminuye considerablemente.

Es importante mencionar que existió disolución de pirita debido a las extracciones de hierro obtenidas, las cuales fueron superiores al $100 \%$ (Fig. 12). Esto es un efecto no deseado ya que se estaría perdiendo masa del aditivo catalizador.

En el análisis realizado en QEMSCAN a los residuos de las pruebas a $1: 1,1: 5$ de calcopirita:pirita presentados en la figura $13 \mathrm{C}$ y $\mathrm{D}$, se observa una reducción de tamaño importante para la pirita después de la lixiviación (Fig. 13 E). Posiblemente, la disolución de la pirita se deba a las condiciones de trabajo utilizadas, tales como temperatura $\left(50^{\circ} \mathrm{C}\right), \mathrm{pH}(<1,2)$ y potencial de solución $(>640 \mathrm{mV})$.

El mapa de minerales (Fig. 13) confirma lo obtenido por los análisis químicos, donde es posible observar el efecto positivo de la pirita en la disolución de la calcopirita (Fig. 13 B, C y D). En ambos casos (1:1 y 1:5) se observa una disminución importante del tamaño de las partículas de los resi- 


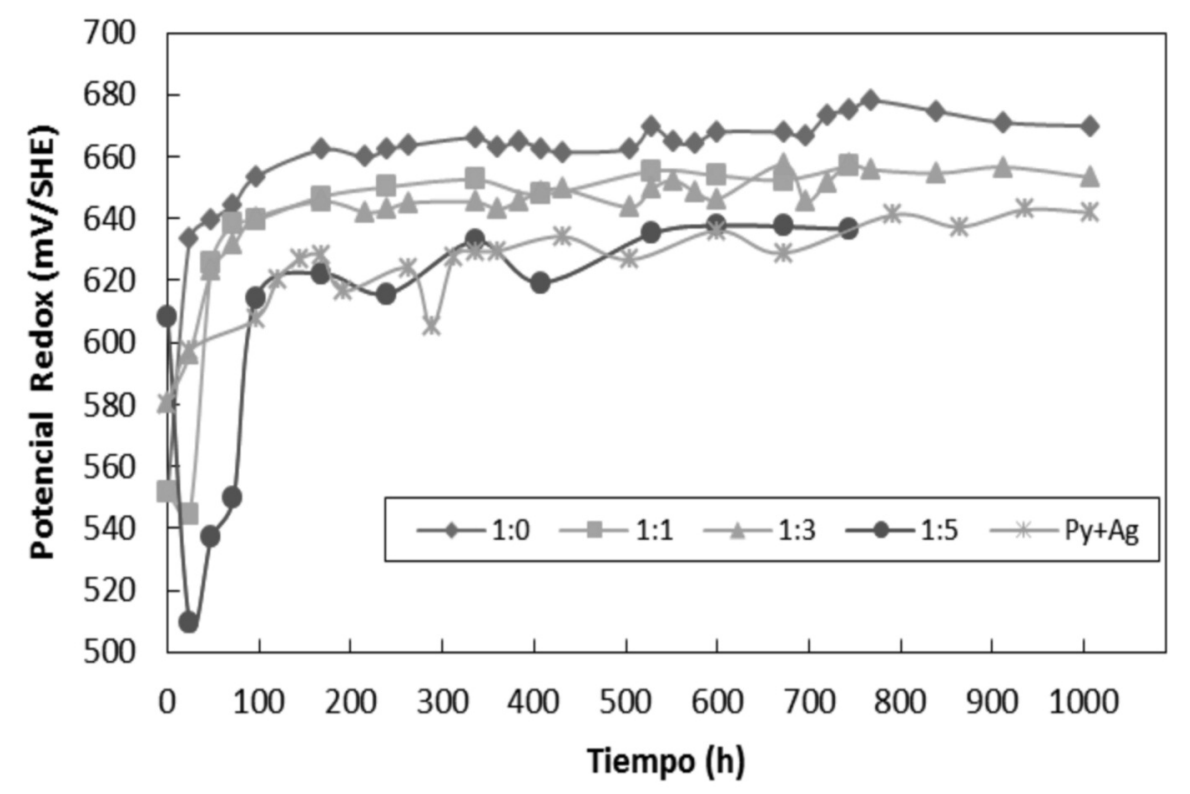

Figura 11. Potenciales de solución de las pruebas realizadas con calcopirita natural en presencia de distintas concentraciones de pirita.

Figure 11. Solution potential of dissolution test from pure chalcopyrite in the presence of pyrite.

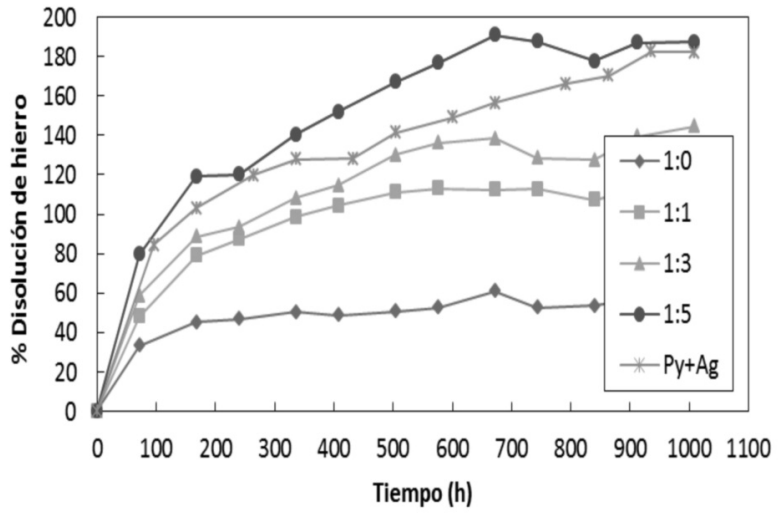

Figura 12. Disolución de hierro de las pruebas realizadas con calcopirita y pirita natural en matraces agitados a $50{ }^{\circ} \mathrm{C} ; 50 \mathrm{~g} / \mathrm{L}$ de $\mathrm{Cl}^{-} ; 0,5 \mathrm{~g} / \mathrm{L}$ de $\mathrm{Cu}^{2+}$ y $0,2 \mathrm{M} \mathrm{H}_{2} \mathrm{SO}_{4}$.

Figure 12. Iron dissolution from chalcopyrite natural with presence of pyrite test in flasks at $50{ }^{\circ} \mathrm{C} ; 50 \mathrm{~g} / \mathrm{L}$ of $\mathrm{Cl}^{-} ; 0.5 \mathrm{~g} / \mathrm{L}$ of $\mathrm{Cu}^{2+}$ and $0.2 \mathrm{M} \mathrm{H}_{2} \mathrm{SO}_{4}$.

duos con respecto a las de alimentación (Fig. 13 A, C y D)

Otra observación importante es que desde los análisis realizados en QEMSCAN se pone de manifiesto la presencia de sulfuros de cobre en los bordes de las partículas de calcopirita, como se puede ver en el mapa de minerales de los residuos (Fig. 13 B, C y D). Pero es interesante que, a pesar de la presencia de estas especies en los bordes de la calcopirita, éstas no se encuentran cubriendo la superficie del mineral de tal forma que no permita el contacto de los reactantes con las partículas de este sulfuro, existiendo bastantes regiones liberadas. Debido a esto, a pesar de los altos potenciales de solución alcanzados, no se observa una clara evidencia de la presencia de alguna capa que sea la responsable de la disminución de la velocidad de disolución de la calcopirita. Para poder afirmar lo recién mencionado es necesario realizar un estudio utilizando otra técnica de análisis ya que es posible que la capa no haya sido percibida debido al límite de detección del equipo. Además, para saber específicamente a qué sulfuro de cobre corresponde, se recomienda realizar estudios utilizando DRX. Esto nos permitiría confirmar o descartar el mecanismo sugerido por varios autores ${ }^{[5,10}$ y 11$]$, quienes aseguran la formación de $\mathrm{CuS}$ como un producto intermedio de la disolución de la calcopirita.

Se observó la presencia de otra especie en los bordes de las partículas de calcopirita la cual no está bien definida. El equipo la detectó como molibdenita pero, a causa del aumento de masa de esta especie en los residuos, se cree que se debe a azufre proveniente de la disolución de la calcopirita. Además, debido a las características del funcionamiento del equipo y a la experiencia de otras investigaciones, 


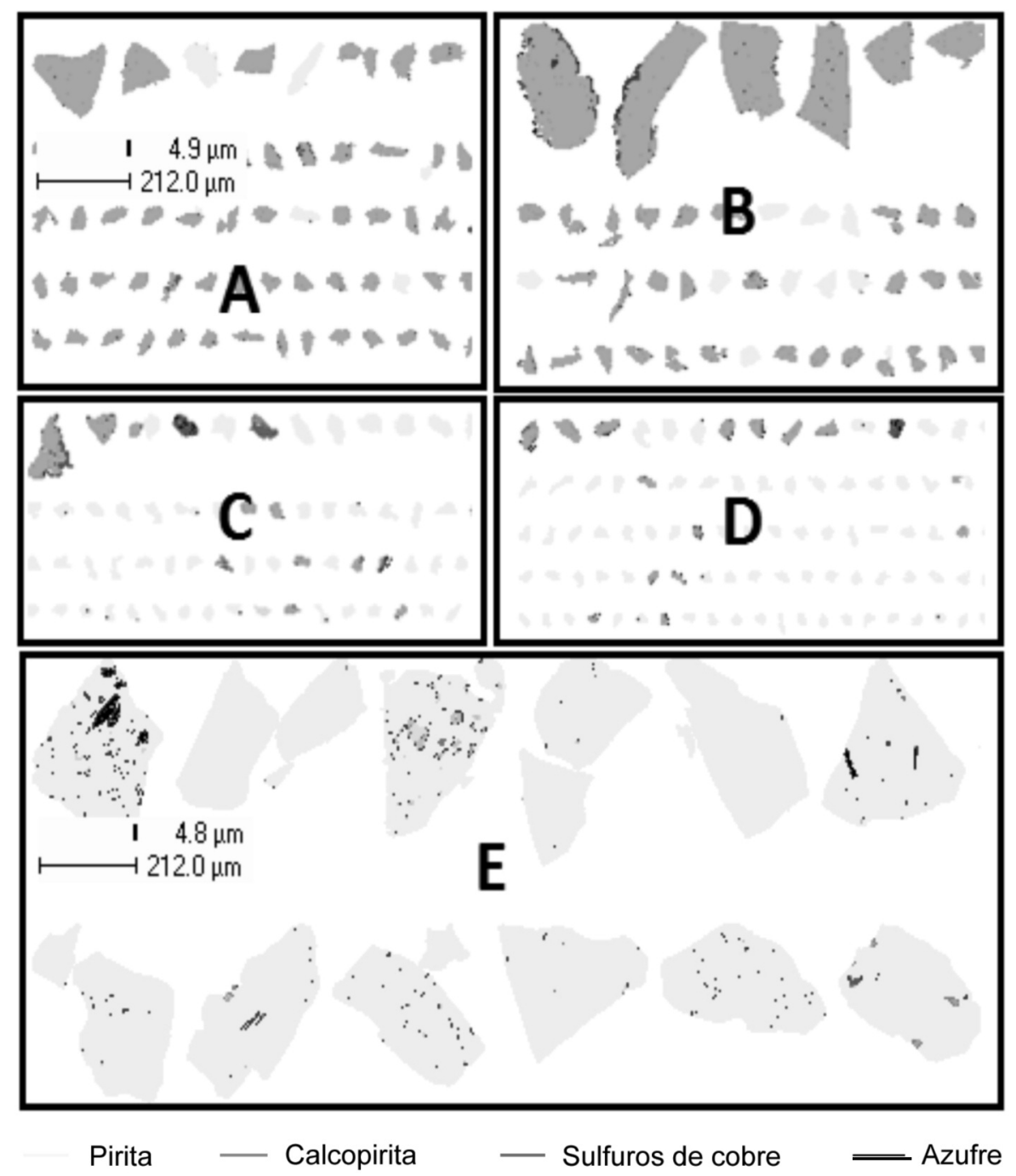

Figura 13. Mapa de minerales donde se observa A: Muestra de calcopirita natural; B: Residuos sin aditivos; C: Residuos prueba 1:1; D: Residuos 1:5; E: Muestra de pirita natural.

Figure 13. Partial mineral maps showing A: Natural chalcopyrite sample; $B$ : Residues of test without additives; C: Residue test 1:1; D: Residue test 1:5; E: Natural pyrite sample.

es sabido que ambas especies pueden confundirse entre sí.

\subsection{Iones de plata (I)}

Se puede observar en la figura 14 que la presencia de estos iones posee un efecto catalizador sobre la disolución de cobre y en presencia de pirita este efecto es aún mayor (Prueba $\mathrm{Py}+\mathrm{Ag}$ que contiene una razón

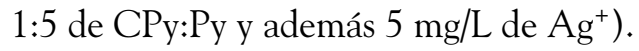

Pareciera que en un medio sin la presencia de iones cloruros (sólo ácido sulfúrico) $(\mathrm{Py}+\mathrm{Ag})$ que contiene una razón 1:5 de CPy:Py y 2 ppm de $\mathrm{Ag}^{+}$ el efecto catalizador es mayor debido a la tendencia de la curva. Esto se asocia a que, en medios clorurados los iones de plata forman complejos fuertes y precipitados con los iones de cloruro, por lo que existe menos ión plata disponible para catalizar la reacción; en cambio en medios sulfatados esto no ocurre, por lo que existe mayor cantidad de iones de plata disponibles. Más adelante será explicado mediante la termodinámica del sistema.

Similar a las pruebas con pirita como aditivo, se observó disolución de pirita para las pruebas que contenían este mineral y esto se puede asociar a los altos 


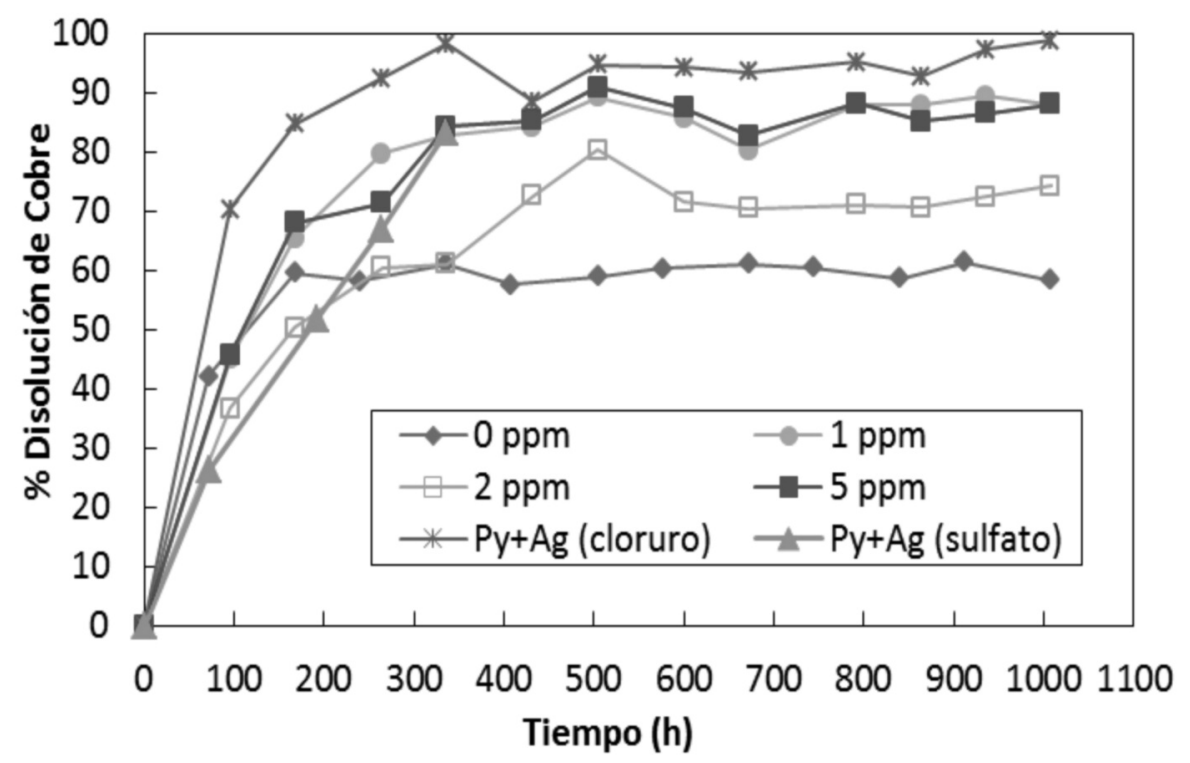

Figura 14. Disolución de cobre a partir de pruebas realizadas con calcopirita natural en presencia de distintas concentraciones de iones de plata realizadas en matraces agitados a $50^{\circ} \mathrm{C} ; 50 \mathrm{~g} / \mathrm{L}$ de $\mathrm{Cl}^{-}-; 0,5 \mathrm{~g} / \mathrm{L}$ de $\mathrm{Cu}^{2+}$ y $0,2 \mathrm{M} \mathrm{H}_{2} \mathrm{SO}_{4}$.

Figure 14. Copper dissolution from natural chalcopyrite with presence of silver ions in flasks at $50{ }^{\circ} \mathrm{C} ; 50 \mathrm{~g} / \mathrm{L} \mathrm{of} \mathrm{Cl}^{-} ; 0.5 \mathrm{~g} / \mathrm{L}$ of $\mathrm{Cu}^{2+}$ and $0.2 \mathrm{M} \mathrm{H}_{2} \mathrm{SO}_{4}$.

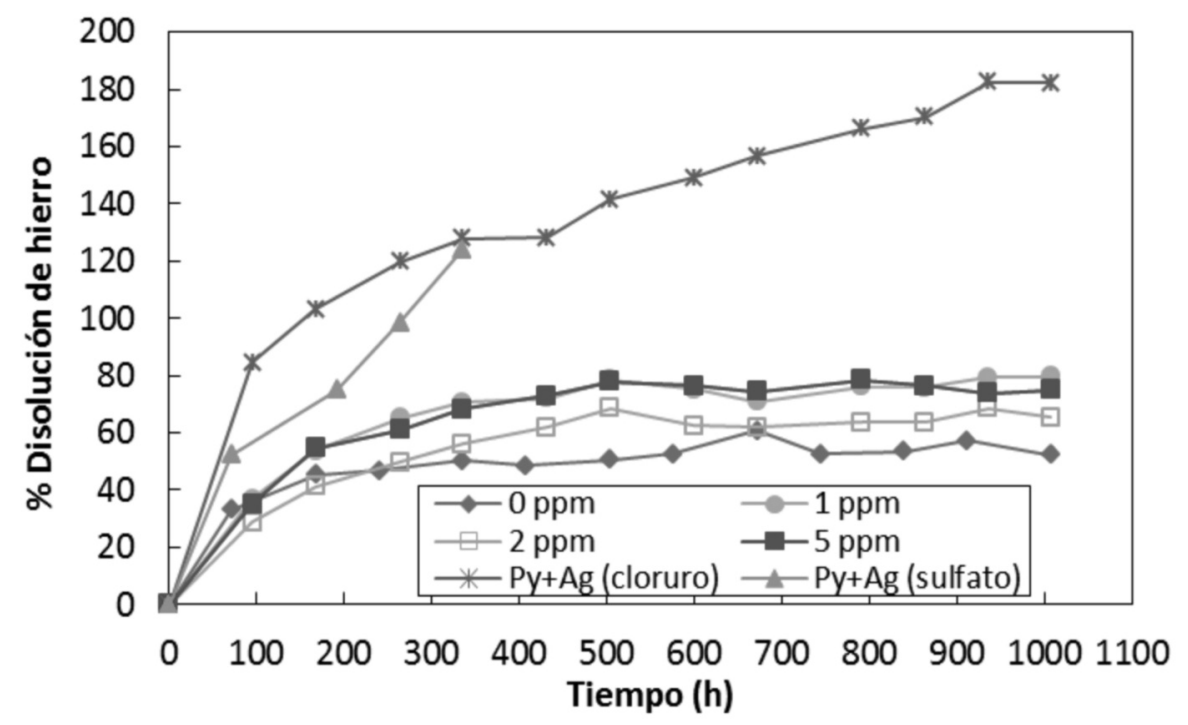

Figura 15. Disolución de hierro a partir de pruebas realizadas con calcopirita natural en presencia de distintas concentraciones de iones de plata realizadas en matraces agitados a $50{ }^{\circ} \mathrm{C} ; 50 \mathrm{~g} / \mathrm{L}$ de $\mathrm{Cl}^{-} ; 0,5 \mathrm{~g} / \mathrm{L}$ de $\mathrm{Cu}^{2+}$ y $0,2 \mathrm{M} \mathrm{H}_{2} \mathrm{SO}_{4}$.

Figure 15. Iron dissolution from leaching tests with pure chalcopyrite with the presence of silver ions in flasks at $50{ }^{\circ} \mathrm{C} ; 50 \mathrm{~g} / \mathrm{L} \mathrm{of} \mathrm{Cl}-; 0.5 \mathrm{~g} / \mathrm{L}$ of $\mathrm{Cu}^{2+}$ and $0.2 \mathrm{M} \mathrm{H}_{2} \mathrm{SO}_{4}$. 


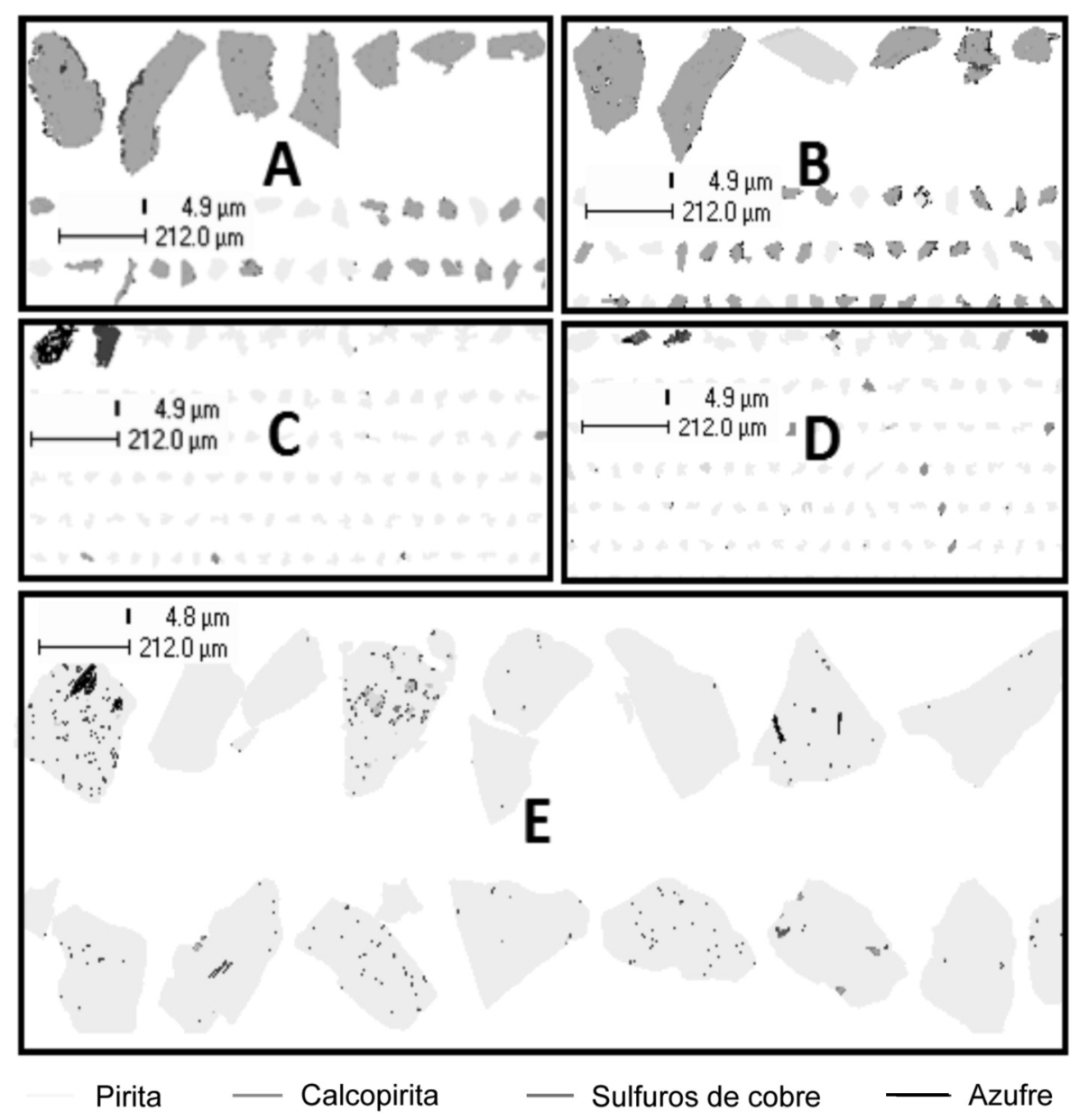

Figura 16. Mapa de minerales que muestra: $A$ : Sin aditivos, $\mathrm{B}: 2 \mathrm{ppm} \mathrm{Ag}{ }^{+}, \mathrm{C}: 1: 5+5 \mathrm{ppm} \mathrm{Ag}+$ (cloruro), D: 1:5 + $2 \mathrm{ppm} \mathrm{Ag}^{+}$(sulfato), E: Pirita natural.

Figure 16. Partial mineral maps showing: A: Without additives, B: 2 ppm $\mathrm{Ag}^{+}, \quad$ C: $1: 5+5 p p m \mathrm{Ag}^{+}$(chloride media), $D: 1: 5+2 p p m \mathrm{Ag}^{+}$ (sulphate), E: Pure pyrite.

valores de potencial de solución, a la temperatura y pH (Fig. 15).

Esto también se observó en los análisis de los residuos realizados en QEMSCAN que están presentados en la figura 16, donde claramente existe una disminución importante en el tamaño de partícula de la pirita natural en los residuos (Fig. 16 C, D y E). Además, se observa con claridad la mayor disolución de las pruebas con presencia de pirita e iones de plata tanto en medio ácido como en medio clorurado (Fig. $16 \mathrm{C}$ y D) en comparación con las pruebas sin aditivos y con 2 ppm de $\mathrm{Ag}^{+}$(Fig. $16 \mathrm{~A} \mathrm{y} \mathrm{B).}$

Cabe mencionar que no se detectó la presencia de ningún precipitado de cloruro y sulfuro de plata como han mencionado otros autores ${ }^{[12]}$, probablemente debido al límite de detección del equipo, por lo que no es posible descartar su presencia, no siendo posible entonces confirmar qué mecanismo es el que está ocurriendo.

$\mathrm{Al}$ igual que en las pruebas realizadas con pirita como aditivo, se detectó la presencia de sulfuros de cobre y azufre en los bordes de las partículas de calcopirita.

\subsection{Termodinámica de los iones de plata (I) en medios clorurados a $50^{\circ} \mathrm{C}$}

En las figuras 17, 18 y 19 se presentan los diagramas de especies del sistema $\mathrm{Ag}$ (I)-cloruro a distintas concentraciones de iones de plata $(1,5$ y $25 \mathrm{mg} / \mathrm{L}$ respectivamente). En las tres situaciones se observa que las especies predominantes son los complejos $\mathrm{AgCl}_{3}{ }^{2-} \mathrm{y}$ 


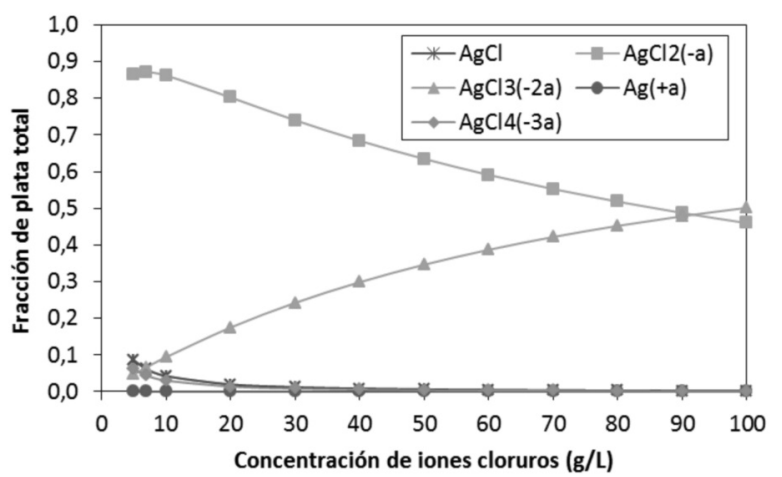

Figura 17. Diagrama de especies del sistema $\mathrm{Ag}(\mathrm{I})-\mathrm{Cl}^{-}$a $50{ }^{\circ} \mathrm{C}$ y $1 \mathrm{mg} / \mathrm{L}$ de $\mathrm{Ag}^{+}$.

Figure 17. Species distribution $\mathrm{Ag}(\mathrm{l})-\mathrm{Cl}^{-}$system at $50{ }^{\circ} \mathrm{C}$ and $1 \mathrm{mg} / \mathrm{L}$ of $\mathrm{Ag}^{+}$.

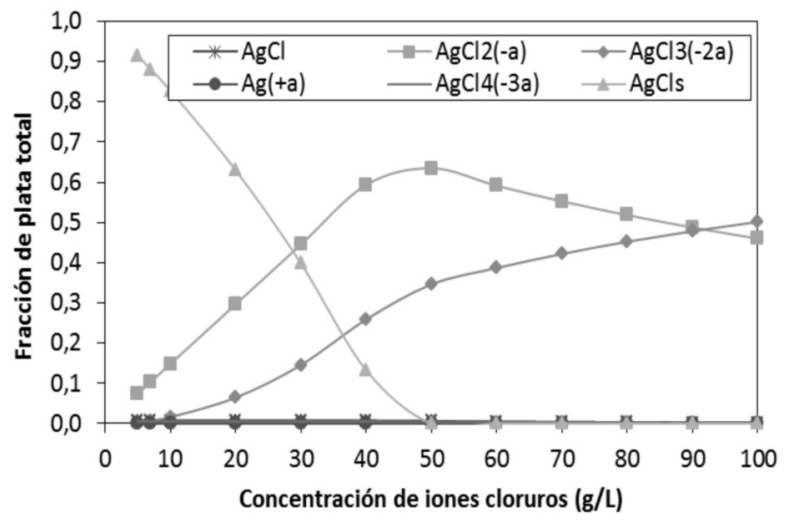

Figura 19. Diagrama de especies del sistema $\mathrm{Ag}(\mathrm{I})-\mathrm{Cl}^{-}$a $50^{\circ} \mathrm{C}$ y $25 \mathrm{mg} / \mathrm{L} \mathrm{de} \mathrm{Ag}^{+}$.

Figure 19. Species distribution $\mathrm{Ag}(\mathrm{I})-\mathrm{Cl}^{-}$system at $50{ }^{\circ} \mathrm{C}$ and $25 \mathrm{mg} / \mathrm{L}$ of $\mathrm{Ag}^{+}$.

$\mathrm{AgCl}_{2}{ }^{\prime}$, además del precipitado $\mathrm{AgCl}$ cuya presencia aumenta al aumentar la cantidad de $\mathrm{Ag}^{+}$en el sistema.

Según algunos autores, el efecto catalizador de los iones de plata en la disolución de la calcopirita en un medio clorurado es menos efectivo en comparación con un medio sulfatado ya que por lo observado en los diagramas de especies, este ión se encuentra principalmente como complejo, por lo que existen menos iones $\mathrm{Ag}^{+}$disponibles para la reacción. Según los resultados obtenidos en nuestros experimentos de lixiviación con calcopirita natural, esto es correcto, ya que claramente fue más rápida la disolución de la calcopirita en un medio sulfatado en comparación con la obtenida en un medio clorurado. Además cabe mencionar que, bajo las concentraciones de iones cloruros utilizados en el presente estudio, no se espera que exista la presencia de $\mathrm{AgCl}(\mathrm{s})$ según lo indican

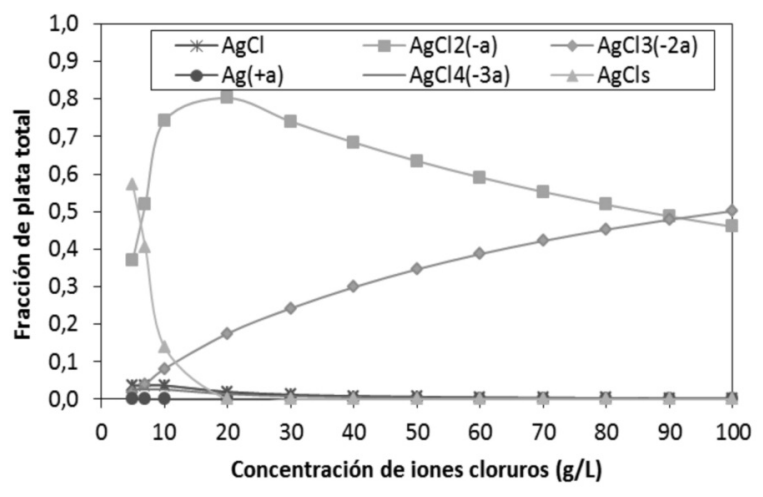

Figura 18. Diagrama de especies del sistema $\mathrm{Ag}(\mathrm{I})-\mathrm{Cl}^{-}$a $50{ }^{\circ} \mathrm{C}$ y $5 \mathrm{mg} / \mathrm{L} \mathrm{de} \mathrm{Ag}^{+}$.

Figure 18. Species distribution $\mathrm{Ag}(\mathrm{l})-\mathrm{Cl}^{-}$system at $50{ }^{\circ} \mathrm{C}$ and $5 \mathrm{mg} / \mathrm{L}$ of $\mathrm{Ag}^{+}$.

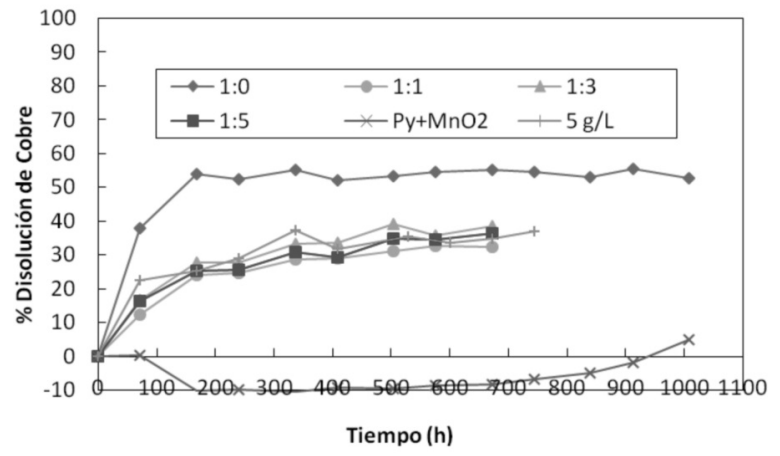

Figura 20. Disolución de cobre a partir de pruebas realizadas en matraces a $50{ }^{\circ} \mathrm{C} ; 50 \mathrm{~g} / \mathrm{L}$ de $\mathrm{Cl}^{-} ; 0,5 \mathrm{~g} / \mathrm{L}$ de $\mathrm{Cu}^{2+}$ y $0,2 \mathrm{M} \mathrm{H}_{2} \mathrm{SO}_{4}$ en presencia de distintas concentraciones de $\mathrm{MnO}_{2}$.

Figure 20. Copper dissolution from tests with pure chalcopyrite in flasks at $50{ }^{\circ} \mathrm{C} ; 50 \mathrm{~g} / \mathrm{L} \mathrm{of} \mathrm{Cl}^{-}$; $0.5 \mathrm{~g} / \mathrm{L} \mathrm{of} \mathrm{Cu}^{2+}$ and $0.2 \mathrm{M} \mathrm{H}_{2} \mathrm{SO}_{4}$ with the presence of $\mathrm{MnO}_{2}$.

los cálculos termodinámicos y esto está de acuerdo con los análisis de QEMSCAN.

\section{4. Óxido de manganeso (IV)}

En la figura 20 se encuentran los porcentajes de cobre disuelto obtenidos para cada experiencia. A partir de éstos, se observa que el $\mathrm{MnO}_{2}$ afecta de forma negativa a la disolución de la calcopirita ya que todas las curvas de disolución se encuentran bajo la prueba sin aditivo excepto para la que contiene pirita, óxido de manganeso (IV) y calcopirita natural en proporciones 1:5:5, donde la lixiviación es negativa, por lo 


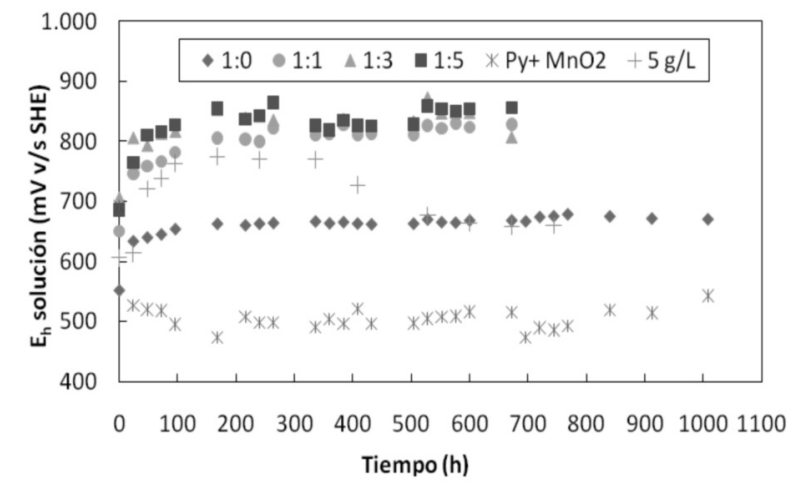

Figura 21. Potencial de solución de las pruebas realizadas en matraces agitados con calcopirita natural en presencia de distintas concentraciones de $\mathrm{MnO}_{2}$.

Figure 21. Variation of solution potential with time for the tests with pure chalcopyrite and presence of $\mathrm{MnO}_{2}$ with different concentrations.

que todo el cobre inicial de la solución de lixiviación $\left(0,5 \mathrm{~g} / \mathrm{L}\right.$ de $\left.\mathrm{Cu}^{2+}\right)$ precipitó.

Esta situación puede ser explicada a través de los potenciales de solución. Como se muestra en la figura 21, los potenciales alcanzados por las pruebas que contienen razones de calcopirita:óxido de manganeso de: 1:1, 1:3, 1:5 y $5 \mathrm{~g} / \mathrm{L}$ de manganeso, sobre los $750 \mathrm{mV}$, provocaron la rápida disminución inicial de la disolución de la calcopirita. Ésta podría ser la razón por la que dichas curvas se encuentran bajo la curva sin la presencia de aditivos, cuyo potencial de solución es mucho menor $(650 \mathrm{mV})$.

Para el caso de la prueba que contenía $\mathrm{CPy}: \mathrm{Py}: \mathrm{MnO}_{2}$, los potenciales disminuyeron a valores bajo $540 \mathrm{mV}$ y el pH aumentó a 4 ( Fig. 22). Esto probablemente provocó que el cobre inicial presente en la solución precipitara y por ende las disoluciones de cobre resultaran negativas.

Además de la precipitación de cobre, también ocurrió la precipitación de hierro debido a que no se detectó este elemento en solución, como se puede observar en la figura 23, y a la tonalidad de los residuos de lixiviación (café rojizo). Esto, al igual que el cobre, está relacionado con los pH de la solución. Otro punto importante de esta prueba es que sólo existió disolución de manganeso, como se puede apreciar en la figura 23.

A partir del mapa de minerales (Fig. 24) se puede corroborar lo mencionado anteriormente. En él se observa la presencia de precipitados de cobre sobre la superficie del óxido de manganeso (IV), el cual pareciera ser atacamita. Esto se debe probablemente a que la forma prismática y los grandes tamaños de las partículas del $\mathrm{MnO}_{2}$ poseen una gran superficie

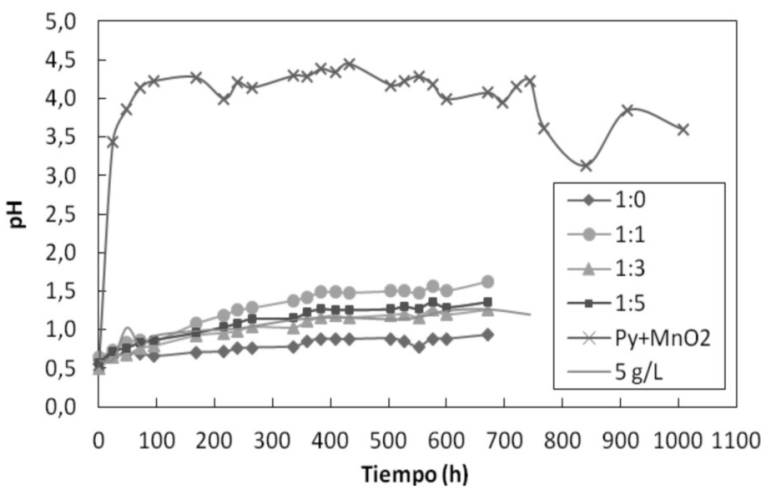

Figura 22. pH de la solución de pruebas realizadas en matraces agitados con calcopirita natural en presencia de distintas concentraciones de $\mathrm{MnO}_{2}$.

Figure 22. Effect of $\mathrm{MnO}_{2}$ on the $\mathrm{pH}$ during dissolution of pure chalcopyrite leached in shake flask.

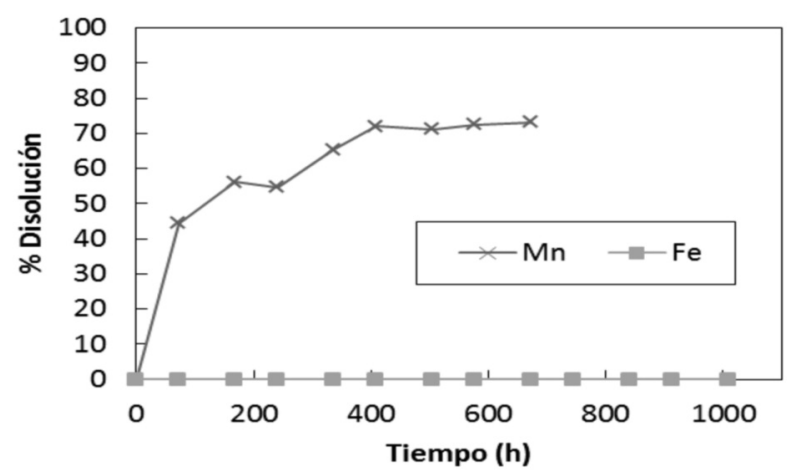

Figura 23. Disolución de hierro y manganeso para la prueba realizada en matraz con calcopirita natural en presencia de calcopirita, pirita y $\mathrm{MnO}_{2}$.

Figure 23. Dissolution of manganese and iron for test with pure chalcopyrite, pyrite and $\mathrm{MnO}_{2}$.

efectiva disponible para la reacción y, además, la gran reactividad de este compuesto lo hace susceptible a la precipitación de especies sobre su superficie, como fue el caso de la atacamita, por lo que teniendo disponible cobre, cloruro, oxígeno e iones de hidrógeno y bajo las condiciones de $\mathrm{pH}$ alcanzadas se formó este precipitado de cobre. Esto resulta muy interesante para futuras investigaciones donde se pueden utilizar especies de este tipo tales como copper wad para la eliminación de impureza a partir de concentrados sucios ya que estos compuestos poseen una superficie porosa la cual es capaz de adsorber muchas especies. 


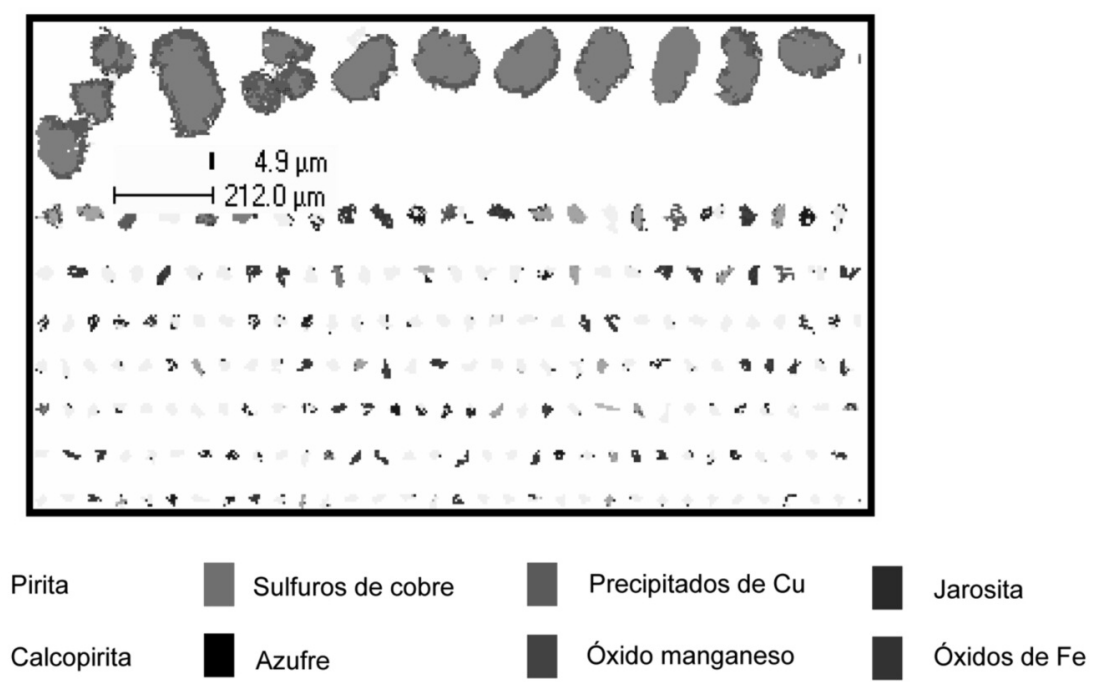

Figura 24. Mapa de minerales de residuos de la prueba realizada con calcopirita, pirita y óxido de manganeso (IV) en proporciones 1:5:5 respectivamente.

Figure 24. Partial mineral maps showing residues for test 1:5:5 to chalcopyrite: pyrite: $\mathrm{MnO}_{2}$ proportion.

Para el caso de precipitados de hierro, en el mapa de minerales se observa la presencia de óxidos de hierro y jarosita. Ambos están localizados en los bordes de las partículas de pirita como se puede observar en la figura 24. Esta situación probablemente se deba a que el $\mathrm{MnO}_{2}$ actuó como un catalizador de la disolución de la pirita y debido a las condiciones de $\mathrm{pH}$ presentes, este hierro disuelto precipitó en la forma de jarosita u óxidos de hierro. Esto también explicaría la alta disolución de manganeso.

Existen autores que han reportado el efecto catalizador del $\mathrm{MnO}_{2}$ en la disolución de la pirita mediante un contacto galvánico, debido a la diferencia de potenciales de reposo entre ambos minerales, donde la pirita se oxida a $\mathrm{Fe}^{2+}$ y el óxido de manganeso (IV) se reduce al ión $\mathrm{Mn}^{2+}$.

\section{Recomendaciones}

Evaluar el efecto de parámetros y aditivos en la cinética de disolución de la calcopirita manteniendo los potenciales de solución constantes dentro del rango 540-630 $\mathrm{mV}$ para observar si posee efectos similares.

Realizar análisis con técnicas más apropiadas para evaluar la existencia de la capa pasivante que mencionan varios autores ya que probablemente en el presente trabajo no fue posible detectarla debido a los límites de detección del equipo.
Realizar análisis con DRX para determinar qué especie de sulfuros de cobre se encuentra en los bordes de las partículas de calcopirita.

\section{CONCLUSIONES}

- La disolución de la calcopirita en medios clorurados es dependiente del potencial de la solución. Cuando los $\mathrm{E}_{\mathrm{h}}$ alcanzan valores entre 540-630 mV (SHE) las velocidades de disolución son aceptables y cuando aumentan sobre este rango, la disolución disminuye considerablemente.

- A pH alrededor de 2,5 la disolución de cobre en reactores es mejorada debido a que a estos valores no existe hierro en solución ya que se forman precipitados de este elemento provocando una disminución de los potenciales de la solución donde la velocidad es aceptable. La presencia de precipitados de hierro bajo las condiciones estudiadas no provocó la detención de la disolución de la calcopirita.

- La disolución de la calcopirita en reactores es afectada positivamente con el aumento de la temperatura entre 35 y $75^{\circ} \mathrm{C}$ con un valor de energía de activación de 96,55 kJ/mol, indicándonos que la velocidad es controlada por la reacción química. A $90^{\circ} \mathrm{C}$ las partículas sufrieron una aglomeración lo cual ocasionó un efecto negativo en la disolución. 
- La pirita y los iones de plata poseen un efecto catalizador en la reacción de disolución del concentrado y calcopirita natural. La presencia de ambos mejora aún más la velocidad de disolución.

- La presencia de calcopirita, pirita y óxido de manganeso (IV) en proporciones de 1:5:5 provocó la precipitación de hierro y cobre debido a la disminución de los potenciales de solución a valores bajo los $540 \mathrm{mV}$ y a un aumento del $\mathrm{pH}$ a 4 .

- El $\mathrm{MnO}_{2}$ posee un efecto negativo en la disolución de la calcopirita natural debido a los altos valores de $E_{h}$ alcanzados ( $>700 \mathrm{mV}$ ) y a la importante diferencia de tamaños de partículas entre el $\mathrm{MnO}_{2}$ y la $\mathrm{CuFeS}_{2}$.

\section{REFERENCIAS}

[1] A.E. Elsherief, Miner. Eng. 15 (2002) 215-223.

[2] M.B Stott, H.R Watling, P.D. Franzmann y D. Sutton, Miner. Eng. 13 (2000) 1.117-1.127.
[3] Z.Y. Lu, M.I. Jeffrey y F. Lawson, Hydrometallurgy 56 (2000) 189-202.

[4] P.A. Moreno, H. Aral, J. Cuevas, A. Monardes, M. Adaro, T. Norgate y W. Bruckard, Miner. Eng. 24 (2011) 852-858.

[5] L. Velásquez, M. Nicol y H. Miki, Hydrometallurgy 103 (2010) 108-113.

[6] E.M. Córdoba, J.A. Muñoz, M.L. Blázquez, F. González y A. Ballester, Hydrometallurgy 93 (2008) 88-96.

[7] J.E. Dutrizac, Hydrometallurgy 29 (1992) 1-45.

[8] M. AL-Harahsheh, S. Kingman y A. AL-Harahsheh, Hydrometallurgy 91 (2008) 89-97.

[9] G. Nazari, D.G. Dixon y D.B. Dreisinger, Hydrometallurgy 105 (2011) 251-258.

[10] L. Velásquez, M. Nicol y H. Miki, Hydrometallurgy 103 (2010) 80-85.

[11] L. Velásquez, M. Nicol y H. Miki, Hydrometallurgy 103 (2010) 86-95.

[12] N. Hiroyoshi, M. Arai, H. Miki, M. Tsunekawa y T. Hirajima, Hydrometallurgy 63 (2002) 257267. 